\title{
Arbeitgeberverbände: Interessenvertretung und Lobbying
}

\author{
Unternehmen werden als Lobbyisten politisch aktiver. Sie verlassen sich nicht mehr auf \\ die Verbände. Die Unternehmen bleiben zwar meist Mitglieder in den Verbänden, sie \\ versuchen aber zunehmend, ihre Interessen jenseits der Verbände lobbyistisch durchzu- \\ setzen. Parallel dazu nimmt die gestaltende Kraft der Arbeitgeberverbände, die bestrebt \\ sind, umfassende und kollektive Positionen zu formulieren, ab. Dies hat Folgen für die \\ Arbeitsmarkt- und Sozialpolitik.
}

RUDOLF SPETH

\section{Einleitung}

Wenn heute nach der Bedeutung der Arbeitgeberverbände gefragt wird, dann muss neben ihrem wirtschaftlichen Gewicht auch immer ihre Funktion im politischen Prozess und im politischen System betrachtet werden (vgl. Speth 2010a). Die Arbeitgeberverbände sind Ausdruck und Ergebnis einer kollektiven Anstrengung der Unternehmen, ihre Stimmen zu bündeln, ihnen Gehör und politischen Einfluss zu verschaffen. Diese Form der kollektiven Handlungsfähigkeit hängt an Voraussetzungen und förderlichen Bedingungen, wie sie sich in der Bundesrepublik über Jahrzehnte hinweg entwickelt haben. Die kollektive Handlungsfähigkeit der Unternehmen ist daher keine feste Größe, sie wird vielmehr spürbar von den Veränderungen beeinflusst, die sich in der inneren und äußeren Umwelt der Arbeitgeberverbände vollziehen. In beiden Bereichen diagnostizieren Beobachter heute deutliche Erosionsprozesse.

Eine Standortbestimmung der gegenwärtigen kollektiven Handlungsfähigkeit der Arbeitgeberverbände erfordert folglich, sowohl ihre aktuelle Stellung im politischen und ökonomischen System in den Blick zu nehmen, als auch nach ihrer Verfasstheit als Mitgliederorganisation zu fragen. Denn es sind die Mitglieder, die die ökonomische Basis des Verbands bilden und die grundsätzlich den Willen zur kollektiven Handlungsfähigkeit aufbringen. Wenn aber die Mitgliedsunternehmen ihren eigenen Verbänden die Un- terstützung entziehen und sich stattdessen selber politisch stärker artikulieren, eigenständig Lobbying betreiben, dann wird die verbandliche Interessenvertretung geschwächt.

Der Beitrag versucht die These zu begründen, dass ein solcher Prozess derzeit zu beobachten ist: Die verbandliche Interessenvertretung der Arbeitgeberverbände wird schwächer, und im Gegenzug wird das Lobbying der Unternehmen, also der Verbandsmitglieder, stärker. Die Ursachen für diese Verschiebungen - so die Arbeitshypothese - liegen im Wandel des politischen und ökonomischen Systems.

Mit dem Begriff des Lobbying wird im Folgenden eine bestimmte Form der Interessendurchsetzung bezeichnet, die sich von der kollektiven, verbandlichen Interessenvertretung unterscheidet. ${ }^{1}$ Der Gegenstand des Lobbying sind eng definierte Interessen, die möglichst vollständig, ohne verbandliche Kompromissbildung durchgesetzt werden sollen. Grundlage des Lobbying sind Nutzenkalkulationen von Partikularinteressen, die sich zu Verteilungskoalitionen zusammenschließen. Ein wesentliches Kennzeichen solcher Koalitionen ist der Versuch, die staatliche (Um-)Ver-

1 In einem weiteren Verständnis bezeichnet "Lobbying“ jede Form der Interessenvertretung und -durchsetzung. In einer solch erweiterten Definition werden allerdings auch die Differenzen zwischen akzeptierten und problematischen Formen der Einflussnahme auf politische Entscheidungen eingeebnet. 
teilung von Gütern, Rechten, finanziellen Mitteln und Vorteilen möglichst optimal im Sinne eigener Vorteile zu beeinflussen. „Das Lobbying kennzeichnet das Bestreben, 'ein möglichst großes Stück vom Kuchen' zu bekommen, ohne selbst zur Lösung übergreifender Fragen beizutragen" (Speth 2010b, S. 11). Der Vorteil des Lobbyingbegriffs besteht darin, dass damit auch die Adressaten, die Methoden und weitere Erscheinungsformen des Handelns von Pressure Groups ${ }^{2}$ ins Augenmerk rücken.

Der Beitrag geht folgendermaßen vor: Im ersten Schritt werden die tradierten Formen der Einbindung der Arbeitgeberbände in das politische System skizziert (Abschnitt 2). Die Politikwissenschaft hat für diese Verschränkungen und Koordination des staatlichen und des verbandlichen Sektors die Heuristik des Korporatismus entwickelt. Die Diagnose einer veränderten oder gar beendeten korporatistischen Einbindung (3) bildet den Ausgangspunkt für den weiteren Gang der Argumentation.

Welche politischen und ökonomischen Wandlungsprozesse das Handeln der Arbeitgeberverbände verändern, zeigt Abschnitt 4. Es sind insbesondere die eigenständiger agierenden Unternehmen, die zu einer neuen politischen Rolle der Unternehmen insgesamt führen. Das wiederum erfordert, dass sich die Unternehmen auch für veränderte politische und mediale Kommunikationsformen öffnen was letztlich auch ihre Performance (erneut) beeinflusst.

Abschnitt 5 illustriert die Strategien und Instrumente, mit denen die Arbeitgeberverbände heute ihre Interessen im politischen Prozess und gegenüber den Medien und der Gesellschaft zur Geltung bringen. Die veränderte politische Kommunikation und Interessenvertretung in der Mediengesellschaft wird anhand der Initiative Neue Soziale Marktwirtschaft (INSM) illustriert. Abschnitt 6 zieht ein Fazit und streicht nochmals die Anforderungen heraus, mit denen die Arbeitgeberverbände in einer globalisierten Ökonomie konfrontiert sind und die es ihnen derzeit erschweren, ihre Interessen und Expertise ähnlich wirksam wie in der Vergangenheit in Entscheidungs- und Normsetzungsgremien einzubringen.

\section{Tradierte Formen der Einbindung}

Der Einfluss der Arbeitgeberverbände auf politische Entscheidungen beruht auf Beziehungsstrukturen formeller und informeller Art, die zusammengenommen die Einbindung der Arbeitgeberverbände in das politische System ausmachen. Diese Verbindungslinien zu den Institutionen und Entscheidungsarenen des politischen Systems sind nach wie vor gegeben und wirksam. Über diese Kanäle üben die Verbände Einfluss aus und wirken nicht nur an der Gestaltung der Arbeitsbeziehungen mit, sondern sind darüber hinaus in die verschiedenen Felder der Sozialpolitik eingebunden (vgl. Speth 2010a).
Die vielfältigen Koordinationsstrukturen zwischen Staat und Wirtschaft in Deutschland wurden mit den Begriffen der korporatistischen Steuerung und des Rheinischen Kapitalismus (vgl. Vitols 2006) verbunden. Beides bezieht sich vor allem auf die für die Wirtschafts- und Arbeitgeberverbände charakteristische Form der Einbindung in das politische System über institutionalisierte Zugänge (Winter 2003). Dies trifft vor allem auf die Arbeitgeberverbände zu, weil diese schon zum Ende des 19. und des beginnenden 20. Jahrhunderts an der Herausbildung der koordinierten Marktökonomie in Deutschland beteiligt waren (vgl. Schroeder/ Weßels 2010). ${ }^{3}$ Die damit verbundenen Steuerungsleistungen beruhen zum einen auf Selbstkoordination und Selbstorganisation und zum anderen auf einer Verschränkung der Arbeitgeberverbände mit dem staatlichen Sektor bzw. sie fußen auf den Zugängen, den die Verbände hierzu haben.

In den sozialpolitischen Feldern von Gesundheit, Rente und Arbeitsmarkt vollzog sich die korporatistische Einbindung auf drei Ebenen: der Tarifautonomie, der betrieblichen Interessenvertretung und der Selbstverwaltung. Trampusch (2009, S. 347) spricht für die Beteiligung an den Strukturen der Selbstverwaltung von einem "genetischen Ausgangspunkt der korporatistischen Einbindung", der heute allerdings nicht mehr dieselbe Wirkungsmächtigkeit wie noch vor zwei oder drei Jahrzehnten hat (vgl. auch Klenk et al. 2012).

Ein zweiter Weg, über den Arbeitgeberverbände im korporatistischen Sinne eingebunden waren, war lange Zeit ihre intensive Beteiligung im Zuge politischer Entscheidungsfindungen. Sie wurden bei der Vorbereitung von Gesetzgebungen oder politischen Richtungsentscheidungen unmittelbar konsultiert. Sie hatten privilegierte Zugänge zu den Ministerialbürokratien und den Arbeitsgruppen in den Parteien und Fraktionen - als Teil eines Elitennetzwerks, das für korporatistische Politik charakteristisch ist und welches das Fundament für die Qualität output-orientierter Steuerungsleistungen bildete (Streeck 2006). Der Korporatismus kann als Elitenkartell verstanden werden und funktionierte solange gut, wie sich die Eliten auf beiden Seiten als gleichrangig anerkannten. Dieses Netzwerk erodiert jedoch mit der veränderten Zusammensetzung der politischen und ökonomischen Eliten. Je stärker die in internationalen Unternehmen sozialisierte Managergeneration und je dünner die

2 Mit diesem Begriff, der aus der Pluralismustheorie stammt, werden häufig Interessengruppen bezeichnet, weil es ihnen vor allem darum geht, Druck auf politische Entscheidungen auszuüben.

3 Konstitutiv für diese institutionelle Struktur sind auch die Gewerkschaften, denn die Stärke und Intensität der institutionellen Einbindung hängt immer auch von den komplementären Organisationen ab. Die Arbeitgeberverbände werden daher auch als "Gegenverbände" (Leckebusch 1966) der Gewerkschaften beschrieben. Dieses Verhältnis hat insbesondere für die Gestaltung der Arbeitsbeziehungen konstitutive Bedeutung. 
personale Verbindung zwischen Arbeitgeberverbänden, Gewerkschaften und Politik wurde, desto schwieriger gestalten sich korporatistische Aushandlungsprozesse.

\section{Veränderung der Staat-Verbände- Beziehung}

Beobachter diagnostizieren seit Mitte der 1990er Jahre eine Veränderung in der Einbeziehung der Verbände in das politische System, die mit dem Begriff "Post-Korporatismus“ (Streeck 2006, S. 173) oder als Entkopplung von Staat und Verbänden beschrieben wird (Trampusch 2005; Höpner 2007; Klenk et al. 2012). Festgestellt wird ein Wandel vom Korporatismus hin zum Pluralismus, der mit einer veränderten Stellung der Verbände begründet wird. Für diese Veränderung gibt es mehrere Gründe. Hier ist erstens die Autonomisierung der Politik zu nennen. Darunter wird verstanden, dass sich in der Politik ein Elitenwechsel vollzieht, in dessen Folge sich die Rekrutierung des politischen Personals verändert. Politiker können heute seltener als in der Vergangenheit auf eine Verbandskarriere verweisen. Im Gegenzug sitzen auch weniger Verbandsvertreter in den Parlamenten. Die Verbandsfärbung des Deutschen Bundestags hat in den letzten Legislaturperioden signifikant abgenommen (vgl. Speth 2010a). Zweitens verändert sich die Beziehung der Verbände zu den Parteien. Auch hier zeigt sich ein Wandel der Elitenrekrutierung sowie eine Verringerung von „overlapping memberships“. Es gibt zwar noch die traditionelle Nähe der Gewerkschaften zur SPD und der Unternehmerverbände zu CDU und FDP, doch die Verbindungen lockern sich: Die Mittelstands- und Wirtschaftsvereinigung in der CDU hat rund 28.600 Mitglieder; nur zwei Mitglieder sind zurzeit (August 2013) Abgeordnete im Deutschen Bundestag. Auch in den anderen Parteien gibt es Vereinigungen des Mittelstandes (vgl. Krickhahn 2010). Insgesamt aber ist das politische $\mathrm{Ge}-$ wicht dieser Vereinigungen, verglichen mit den Mittelstandsverbänden, eher gering.

Beobachten lässt sich des Weiteren eine Autonomisierung der Politik in verschiedenen sozialpolitischen Feldern. Sie drückt sich darin aus, dass der Staat Regelungs- und Politikkompetenz wieder stärker an sich zieht. Dies war etwa bei der Gesundheitsreform 2007 der Fall und lässt sich auch in den Reformen des Arbeitsmarktes der rot-grünen Koalition beobachten. In früheren Phasen wurde die Formulierung der politischen Vorhaben vielfach an die Verbände delegiert, die Politik legitimierte diese Vorhaben und setzte sie in Gesetzgebung um. Heute beanspruchen politische Akteure mehr Regelungskompetenz, um Märkte entstehen zu lassen, über die dann politische Ziele erreicht werden sollen (vgl. auch Trampusch 2009). ${ }^{4}$ Die Verbände sind zwar nach wie vor in den politischen Prozess eingebunden, die politischen Akteure reklamieren aber zunehmend für sich die inhaltliche Gestaltungskompetenz.
Insgesamt rücken die Verbände, und insbesondere die Arbeitgeberverbände, immer mehr in eine lobbyistische Position. Im selben Maße wie ihr Einfluss in politischen Entscheidungsprozessen erodiert und ihre Kraft nachlässt, unterschiedliche Unternehmensinteressen zu aggregieren, um einen branchenweiten Konsens herzustellen, wird die Vertretung von Interessen situativer und weniger umfassend. Damit aber geraten die Verbände in Konkurrenz zu den eigenen Mitgliedern und anderen verbandlichen Interessengruppen.

\section{Unternehmen als politische Akteure}

Der Trend, dass Unternehmen zunehmend politisch eigenständig agieren und damit auch die skizzierte Konkurrenz zwischen Verbänden und Einzelunternehmen wächst, verläuft nicht gleichförmig: Bei Großunternehmen ist eine solche Entwicklung schon lange zu beobachten, während kleine und mittlere Unternehmen nur gelegentlich eigene politische Aktivitäten entfalten und mehrheitlich nach wie vor auf die Verbände vertrauen.

Das veränderte politische Aktivitätspotenzial der (Groß-)Unternehmen hat mehrere Gründe. Sie eröffnen sich damit erstens größeren Spielraum bei der Interessendurchsetzung. Sie bleiben zwar Mitglied im Verband, setzen aber in spezifischen Fällen auf das individuelle Lobbying. Weil die Verbandsposition immer eine Kompromissbildung ist, mit der ein Branchenkonsens erzielt werden soll, steigt für wirkungsmächtige Unternehmen die Neigung zum Lobbying, wenn damit Wettbewerbsvorteile erreicht werden können. Zum Zweiten hat die Bereitschaft, Verbandspositionen anzuerkennen, nachgelassen. Dies hängt auch mit der neuen Managergeneration und ihrer anderen Art der Unternehmensführung zusammen. Dominierende Paradigmen wie verstärkte Kostenkontrolle, die Verbesserung der Wettbewerbsposition und die wachsende Konkurrenz sind hier als Antriebsmomente zu nennen. Es ist aber auch die Haltung der neuen Generation von Unternehmensführern, die den bundesrepublikanischen Verbandsstrukturen weniger Bedeutung beimisst, auch weil sie in einer anderen politischen Kultur sozialisiert worden sind. Drittens kommen ökonomische Veränderungen als Gründe hinzu.

4 Allerdings gibt es auch Beispiele für gegenläufige Bewegungen. Das Phänomen der schnellen wirtschaftlichen Erholung nach dem Einbruch der Wirtschaft durch die Finanzmarktkrise 2008 wird mit dem Begriff des "Krisenkorporatismus" (Urban 2010) erklärt. Das Zusammenwirken der korporatistischen Akteure (Staat, Gewerkschaften, Arbeitgeberverbände) ermöglichte die Kurzarbeiterregelung mit der Beschäftigte in den Unternehmen gehalten werden konnten. Damit konnte die Produktion mit der anzie henden Nachfrage schnell wieder hochgefahren werden (Bogedan 2012). 
Viele Unternehmen, die in Deutschland tätig sind, haben ihren Hauptsitz im Ausland. Für sie sind die hiesigen Verbände nur ein Weg unter mehreren, die eigenen Interessen durchzusetzen. Die Interessenpositionen der Unternehmen differenzieren sich jedoch auch im Zuge des globalen Wettbewerbs aus - dem sich mittlerweile auch kleine und mittlere Unternehmen ausgesetzt sehen. Insgesamt werden die sozialpolitischen und arbeitsmarktspezifischen Interessen von Unternehmen nicht mehr in dem Maße durch die Verbände gebündelt, wie dies früher der Fall war. Paster (2010, S. 344) stellt hierzu fest, dass „Interessenkonflikte häufiger auftreten," die Arbeitgeberverbände aber nach wie vor eine wichtig Stimme im sozialpolitischen Feld haben.

Die Gründung von Unternehmensrepräsentanzen (Priddat/Speth 2007), die Beauftragung von Public-Affairs-Agenturen und das Entstehen eigener Public-Affairs-Abteilungen (Siedentopp 2010) veranschaulichen, dass Unternehmen zunehmend als politische Akteure auftreten. Dies gilt ganz besonders für große Unternehmen, die für ihren Erfolg vermehrt in „Nichtmarktstrategien “ ${ }^{5}$ investieren und diese nicht mehr primär an Verbände delegieren. Der Unternehmenserfolg wird zunehmend auch über die Beeinflussung der politischen Regulierung von Märkten gesucht, wobei die Verbandsmitgliedschaften zwar nach wie vor eine wichtige Funktion haben, jedoch nicht mehr allein maßgebend sind.

Des Weiteren lässt sich eine Unzufriedenheit mit der Arbeit der Verbände feststellen, die dazu führt, dass Unternehmen einerseits häufiger Kontakt mit Verbänden haben als gewünscht und sie andererseits an den Verbänden vorbei selbst politisch aktiv werden. Beklagt wird vielfach auch die nationale Beschränkung von Verbänden, die aus ihrer Historie resultiert. Unternehmen sind mittlerweile aber auf vielen Märkten weltweit präsent und Großunternehmen koordinieren ihre Public-Affairs-Strategie für diese Märkte selbst. Zudem wird die Ebene der Europäischen Union aufgrund der wachsenden Europäisierung der Politik bedeutsamer. ${ }^{6}$

Hinzu kommt, dass vonseiten der Politik die spezielle Expertise von Unternehmen erwünscht wird, wenn nicht nur die Branchenmeinung gefragt ist, die häufig als der kleinste gemeinsame Nenner gilt. Unternehmen können dann einen Beitrag zu konkreten Inhalten der Gesetzgebung leisten und gleichzeitig ungefiltert ihre Interessen vertreten. An einigen
Fällen wurde in der Vergangenheit auch deutlich, wie Unternehmen ihre Position, die nicht mit dem Verband abgestimmt war, an die Politik vermitteln und somit den Verband und die Konkurrenten in Zugzwang bringen. ${ }^{7}$ Teilweise gründen Unternehmen einen eigenen Verband, um ihre Interessen wirkungsvoller vertreten zu können. ${ }^{8}$ Im Pharmabereich wiederum hat sich der Bundesverband der Pharmazeutischen Industrie (BPI) aufgespalten, weil kleinere Gruppen, wie die forschenden Arzneimittelhersteller (VfA), glauben, auf diese Weise ihre Interessen wirksamer vertreten zu können. Unternehmen können auch temporäre Allianzen bilden und verfügen damit über ein weiteres Instrument, um neben dem Verband eigenes Lobbying zu betreiben. Diese Form ist relativ kostengünstig und umgeht die Nachteile verbandlicher Kompromissbildung, ohne dabei den Vorteil kollektiver Interessenformulierung aufzugeben. Zudem ist mit diesem Instrument zeitliche und inhaltliche Flexibilität verbunden.

\section{Lobbying}

\subsection{Strategien und Instrumente}

Die Arbeitgeberverbände stehen heute vor der Herausforderung, die sozial- und arbeitsmarktpolitischen Interessen nicht nur gegenüber der Politik, sondern auch in einer Gesellschaft vertreten zu müssen, die sich immer stärker zu einer Mediengesellschaft entwickelt. Im Folgenden werden die Lobbyinginstrumente und -strategien dargestellt, auf die Arbeitgeberverbände im Sinne ihrer Interessenartikulation und -durchsetzung zurückgreifen. Die Interessenvertretung gegenüber den Tarifpartnern wird hier nicht thematisiert. Gegenüber den Gewerkschaften spielen Lobbyinginstrumente kaum eine Rolle; in den tarifpolitischen Aushandlungsprozessen gelten eigene Regeln und Verfahren.

Zunächst ist zwischen politischen und medialen Strategien zu unterscheiden. Die Arbeitgeberverbände sind Organisationen, denen es vor allem darum geht, mit dem für sie relevanten Teil ihrer Umwelt, mit dem politischen Entscheidungszentrum und den Gewerkschaften, zu kom-
5 Das Gegenteil ist die Marktstrategie, bei der es um konkurrenzfähige Produkte und Kundenzufriedenheit geht (Siedentopp 2010, S. 266).

6 Diese ist nicht in allen Politikfelder gleich stark, sondern variiert (vgl. Töller 2008). Das europäische Engagement von Verbänden und Unternehmen richtet sich deshalb auch nach dem Europäisierungsgrad des Politikfeldes, in dem sie tätig sind.

7 Am Fall des Versicherungsunternehmens Allianz kann dies am Beispiel der Gesundheitsreform 2007 deutlich gemacht werden. Die Allianz hatte gegenüber der Politik den Vorschlag gemacht, die Privaten Krankenkassen an den
Kosten der Reform zu beteiligen. Das Angebot bestand darin, eine Versicherungssteuer in der Höhe von 9,5\% auf die Beiträge der Versicherten einzuführen. Dieses Angebot wurde vom Verband der Privaten Krankenversicherungen (PKV) und von den anderen 48 Mitgliedern des Verbandes zurückgewiesen (http://www.rp-online.de/politik/deutschland/allianz-chef-bietet-finanzielle-beteiligung-an-gesundheitsreform-an-1.2317050)

8 Ein Beispiel dafür ist der Bundesverband der Deutschen Sicherheits- und Verteidigungsindustrie e.V. (BDAV), der 2010 gegründet wurde. Er ersetzt den Ausschuss Verteidigungswirtschaft im BDI und hat 47 Mitglieder. 
munizieren. Demgegenüber waren die Gesellschaft und die medial vermittelte Öffentlichkeit für die Ziele der Arbeitgeberverbände lange Zeit von geringerer Bedeutung (vgl. Steiner/Jarren 2009). Entsprechend dürftig waren daher auch die Kapazitäten für mediale Kommunikation ausgebildet. Außer sporadischen Presseerklärungen zu ausgewählten Themen gab es wenig politische Kommunikation mit den Medien und der Öffentlichkeit (vgl. Hoffjann/Stahl 2010; Koch-Baumgarten 2010). Dies hat sich zwar in den letzten Jahren verändert, allerdings nicht in der Art und Weise, dass die Arbeitgeberverbände neue Organisationskapazitäten für PR bereitgestellt hätten. Vielmehr wurde die medienvermittelte Kommunikation an spezielle Agenturen „outgesourced“ bzw. es wurden neue Formen geschaffen, mit denen die Kampagnenführung jenseits verbandlicher Bürokratien möglich wurde und die zudem experimentellen Charakter annehmen konnten. Die entwickelten Aktivitäten zielen in zwei unterschiedliche Richtungen: Zum einen richten sie sich in einer internen Perspektive auf die eigenen Mitglieder. Ihnen sollen die Aktivitäten des Verbandes verdeutlicht und ihre Loyalität soll gestärkt werden. Zum anderen zielen die Aktivitäten auf die öffentliche Meinung, um darüber Einfluss auf das politische Entscheidungszentrum auszuüben.

Mit der Initiative Neue Soziale Marktwirtschaft (INSM) haben die Arbeitgeber - und vor allem Gesamtmetall als größter Verband im Bundesverband deutscher Arbeitgeber (BDA) - einen neuen Weg der lobbyistischen Beeinflussung der Mediengesellschaft beschritten (vgl. Speth 2004; Höfer 2010). Die INSM wurde 1999 zu einer Zeit gegründet, als der gesellschaftliche Diskurs durch ein neoliberales Klima geprägt war. Die Politik der Blair-Regierung in Großbritannien und die amerikanische Wirtschafts-, Fiskal- und Geldpolitik zeigten, dass mit dieser Richtung Erfolge bezüglich Wirtschaftswachstum und gesellschaftspolitischer Gestaltung errungen werden konnten. In dieser Zeit stellte Gesamtmetall einen Betrag von 100 Mio. $€$ für einen Zeitraum von zehn Jahren zur Verfügung, um auf neuartige Weise Themen der Arbeitgeber jenseits der Tarifpolitik in der Mediengesellschaft zu kommunizieren. Die konzeptionelle Verantwortung oblag der Kommunikations- und Werbeagentur Scholz+Friends.

Nach mehr als einem Jahrzehnt lässt sich heute die Strategie, die die Arbeitgeber mit der INSM verfolgen, präziser beurteilen. Die Arbeitgeber finanzieren die INSM nach wie vor weiter. Dies lässt vermuten, dass die Arbeitgeberverbände die Notwendigkeit erkannt haben, jenseits der verbandlichen Lobbyingkanäle, die in der Regel diskret und jenseits der öffentlichen Aufmerksamkeit genutzt werden, die mediale Kommunikation zu stärken. Nach wie vor prägend ist die Aufgabentrennung der Bereiche: Gesamtmetall macht Tarifpolitik, während die INSM zu allgemeinen wirtschafts- und sozialpolitischen Fragen Stellung nimmt und wirtschafts- und gesellschaftspolitische Deutungsangebote aus Unternehmersicht in die Mediengesellschaft kommuniziert. Während Gesamtmetall mehr oder minder ganz ohne mediale Kommunikation auskommt, setzt die INSM dagegen ausschließlich auf dieses Instrument.

Für ihre spezielle Art der politischen Kommunikation hat die INSM ein Format entwickelt, das sich sehr stark an das Portfolio der Public-Affairs-Agenturen anlehnt. „Integrierte Kommunikation“ ist der Schlüsselbegriff, der Werbung, PR, Campaigning, TV-Beiträge, journalistische Arbeit, Social-Media-Aktivitäten und Lobbying umfasst. Die INSM stellt eine Mischung aus politischer Werbeagentur und Think Tank dar.

Der Titel der Initiative „Neue Sozial Marktwirtschaft“ drückt die inhaltliche Stoßrichtung der Kampagne aus. Die Arbeitgeberverbände versuchen mit der INSM das Konzept der Sozialen Marktwirtschaft, das für den Ausgleich von Kapital und Arbeit sowie die Rolle des Staates lange Zeit maßgeblich war, neu zu interpretieren. In dieser Neujustierung werden die Prinzipien der (Markt-)Freiheit, der Selbstverantwortung, der Eigeninitiative, des Wettbewerbs und des schlanken Staats als neue Rahmenbedingungen für die politische und soziale Ordnung propagiert. Dies ist umso bemerkenswerter, als die Arbeitgeberverbände, insbesondere die BDA als Dachverband, zentrale Organisationen sind, die die Ordnung der Sozialen Marktwirtschaft stützen und den Sozialen Kapitalismus verteidigen. Dies kommt insbesondere darin zum Ausdruck, dass sie die sozialstaatlichen Sicherungssysteme anerkennen, sich an Flächentarifverträgen beteiligen und in die Gestaltung der Arbeitsbeziehungen eingebunden sind.

Die Aktivitäten der Wirtschafts- (und nicht nur der Arbeitgeberverbände) zielen auf eine größere Präsenz in der Mediengesellschaft. Im Zuge der INSM wurden mehrere Kampagnen angeschoben, die das Ziel hatten, das Bild der Unternehmen zu verbessern und für eine positive Stimmung in der Bevölkerung zu sorgen. $\mathrm{Zu}$ nennen sind hier die Kampagne „Du bist Deutschland“, die 2005 unmittelbar nach der unerwartet angesetzten Bundestagswahl am 26. September startete und "Deutschland, Land der Ideen“ (vgl. Speth 2006b). Nach der Kampagne „Du bist Deutschland“ folgte eine weitere zum Thema Kinderfreundlichkeit, die allerdings wenig mediale Aufmerksamkeit erzielen konnte.

\subsection{Politische Dienstleister}

Public-Affairs-Agenturen verstehen und inszenieren sich als Trendsetter zeitgemäßer politischer Kommunikation und versuchen damit, Distanz zum etablierten und traditionellen Verbandslobbying zu schaffen. Hoffmann et al. (2007, S. 441) kommen aufgrund von empirischen Befunden zu dem Ergebnis, dass Public-Affairs-Agenturen im Vergleich zu den Verbänden flexibler und ,auf die Wandlungsprozesse politischer Interessenvermittlung unter Bedingungen einer fortschreitenden gesellschaftlichen Verflechtung und der damit einhergehenden Bedeutungszunahme medialer Kommunikation besser eingestellt sind und diese Entwicklungen im eigenen Sinne - etwa durch Professionalisierung der Public Affairs - aktiv zu beeinflussen ver- 
suchen“. Public Affairs kann als Begriff verstanden werden, mit dem die verschiedenen Dimensionen der Kommunikation in technischer und strategischer Hinsicht von Akteuren verbunden werden. Public Affairs umfasst damit Lobbying und mediale Kommunikation. Mit diesem Begriff kann daher die neuere Entwicklung der strategischen Kombination von öffentlicher und nicht-öffentlicher Kommunikation präziser erfasst werden (vgl. Althaus 2005).

Public-Affairs-Agenturen, Kommunikationsagenturen, Lobbyingdienstleister und Anwaltskanzleien sind in den letzten 20 Jahren im Zuge der Professionalisierung der politischen Kommunikation entstanden und können als Unternehmensberatungen im politischen Raum gesehen werden. Das macht sie zugleich zu Konkurrenten der Verbände (hier der Arbeitgeberverbände). Diese Agenturen bieten Dienstleistungen an, die auch die Verbände erbringen. Auf der Ebene des Lobbying umfassen diese Dienstleistungen das Monitoring, die Beobachtung des politischen Prozesses, die Systematisierung der Daten sowie Lobbyingstrategien, Kontakte mit Politikern und Ministerialbeamten, Entwürfe von Gesetzestexten und den Kontakt mit anderen Interessengruppen.

Auf der Ebene der Kommunikation offerieren diese Agenturen ebenfalls eine breite Palette an Dienstleistungen. Durch ihre Verbindung mit den Medien und ihre professionelle Kommunikation in der Mediengesellschaft füllen sie eine Lücke, die durch die Verbände nicht in gleicher Weise zu schließen war (vgl. Koch-Baumgarten 2010; Speth 2010b). Die Public-Affairs-Agenturen machen den Verbänden und ihren Mitgliedern das Angebot einer integrierten Kommunikation, aus der Kunden die passenden Elemente auswählen können. Dieses Angebot umfasst PR, Eventmarketing, Werbung, TV-Beiträge, journalistische Textbeiträge, Dialogmarketing, Profiling, internetbasierte Kommunikation (Social Media, Facebook, Twitter, Google+) und Campaigning. Dienstleister für politische Kommunikation greifen Verbände auf einem weiteren Feld an, das zu den zentralen Verbandsdomänen gehört: den Beteiligungsprozessen. Verbände sind für politische Entscheider und für die Implementation von Politik auch deshalb wichtig, weil sie durch ihre Mitglieder, die sie in Willens- und Meinungsbildungsprozesse involvieren, über eine hohe Legitimation verfügen. Die Dienstleister bieten Ministerien und anderen Institutionen eine Alternative, indem sie Beteiligungsprozesse organisieren. Solche Beteiligungsprozesse durch Agenturen sind wesentlich schneller und effektiver als sie Verbände anbieten können. Somit lassen sich politische Vorhaben schneller implementieren und zudem Legitimation für politische Projekte generieren.

\section{Fazit}

Die Veränderungen, denen sich die Arbeitgeberverbände aber auch alle anderen kollektiven Interessenvertretungs- organisationen aus der Wirtschaft - ausgesetzt sehen, betreffen mehrere Dimensionen (Lang/Schneider 2007). Es ist zu erwarten, dass sich hier in den nächsten Jahren ein beschleunigter Wandel vollzieht, der die Arbeitgeberverbände noch stärker unter Druck setzen wird.

Die Arbeitgeberverbände müssen sich auf andauernde Veränderungen ihrer internen und externen Umwelt einstellen. Die interne Umwelt betrifft vor allem die Mitglieder, das heißt, die Unternehmen. Sie verändern sich weiter - mit und im Zuge des Wandels ökonomischer Strukturen. Längst haben sich viele Unternehmen internationalisiert, während die Verbände immer noch dominant nationale Organisationen sind. Das macht die Verbände weniger attraktiv. Der Anreiz, Mitglied in einem Arbeitgeberverband zu sein, schwindet aber ggf. auch im gleichen Maße wie sich die Interessen der Unternehmen pluralisieren. Dies gilt sowohl für die produktmarktbezogenen wie auch für die arbeitsmarktbezogenen und sozialpolitischen Interessen. Insbesondere die sozialpolitischen Interessen werden von Unternehmen, die ihre Produktion internationalisiert haben, wesentlich vom Export abhängig sind oder gar aus dem Ausland stammen, anders beurteilt als von Unternehmen, die sich dem deutschen Modell des Rheinischen Kapitalismus verpflichtet fühlen. Einige Unternehmen nutzen andere Formen der Interessenvertretung und des Lobbyings jenseits der Verbände, sodass diese sich neuer Konkurrenz gegenübersehen.

Die Veränderungen in der externen Umwelt haben ebenso tief greifende Auswirkungen auf die Arbeitgeberverbände. Der ökonomische Wandel, insbesondere der in Richtung einer Dienstleistungsgesellschaft, lässt das Interessenspektrum breiter werden. Dies betrifft auch die arbeitsmarktspezifischen und insbesondere die sozialpolitischen Interessen. Es lässt sich beobachten, dass Unternehmen aus dem Dienstleistungsbereich sich immer weniger an den sozial- und arbeitsmarktpolitischen Bestand an Regeln halten. Das Ausufern von Niedriglöhnen im Dienstleistungssektor ist hierfür nur ein Beispiel. Dies hängt auch mit der Schwäche des Gegenübers zusammen. Und Letztere reduziert die Notwendigkeit, „Gegenverbände“ zu bilden.

Die Politik in den Dimensionen von politics, polity und policy gehört ebenfalls zu den umweltrelevanten Faktoren für die Arbeitgeberverbände. In der Politics-Dimension sind es Veränderungen in den Beziehungsstrukturen der politischen und verbandlichen Eliten, wodurch die Interessenvertretung stärker lobbyistisch wird. Hinzu kommen die Veränderungen in der Polity-Dimension, das heißt, den institutionellen Strukturen. Die Selbstverwaltung in den sozialen Sicherungssystemen wurde geschwächt, sodass über diesen Weg die Gestaltungsmacht der Arbeitgeberverbände - wie auch der Gewerkschaften - beschnitten wurde.

Auch in der Policy-Dimension, also auf der Ebene der Inhalte insbesondere der Sozialpolitik, haben sich Veränderungen ergeben. Auf vielen Feldern der Sozialpolitik wurde Wettbewerb als neue Steuerungsform eingeführt. Die damit einhergehende Ökonomisierung führt in der Sozial- 
politik zu Verhaltensänderungen und einem stärkeren Gegeneinander der Unternehmen. So hat z.B. die Einführung privater Komponenten in der Altersvorsorge neue Akteure aus den Finanzmärkten in die politische Arena gebracht, was zu einer stärkeren Betonung finanzmarktpolitischer Interessen im System der Altersvorsorge führt.

Auch die Medialisierung stellt für die Verbände eine zentrale Herausforderung dar, denn aus der Vergangenheit herrührend haben sie sich lange als Teil einer nichtöffentlichen Politikarena verstanden. Der Trend in Richtung Mediengesellschaft bedeutet jedoch, dass auch Verbände sich verstärkt der öffentlichen Kommunikation widmen müssen. Dies gelingt nicht allen Verbänden gleichermaßen. Denn dafür ist es notwendig, den Kommunikationsmodus umzustellen, und nicht immer gibt es Kompetenzen und Formate für eine strategische öffentliche Kommunikation in den Verbänden. Vielfach wird diese dann an externe Dienstleister ausgelagert die aber - wie etwa die INSM - gleichzeitig auch eine andere Perspektive in den politischen Diskurs einbringen.

In der Summe schwächen die verschiedenen Veränderungen und die verbandliche Reaktionen die Position der Arbeitgeberverbände. Sie sind stärker auf lobbyistische Politikbeeinflussung angewiesen, weil sie nicht mehr so stark und selbstverständlich dauerhaft an den politischen Prozessen beteiligt sind. Zudem sind Verbände Organisationen, die den Erfordernissen der Mediengesellschaft nur bedingt Rechnung tragen können. Hinzu kommt, dass auf der inhaltlichen Ebene die Sozialpolitik sich stärker einzelwirtschaftlichen Interessen öffnet und kollektive Lösungen entsprechend an Überzeugungskraft verlieren.

\section{LITERATUR}

Althaus, M. (2005): Art. Public Affairs, in: Althaus, M./Geffken, M./Rawe, S. (Hrsg.): Handlexikon Public Affairs, Münster, S. 262-267

Bogedan, C. (2012): Arbeitsmarktpolitik im Spiegel der konjunkturellen Entwicklungen: Die politisch motivierte unterschiedliche Nutzung des Kurzarbeitergeldes 1973-2010, in: Bothfeld, S./Sesselmeier, W./Bogedan (Hrsg.): Arbeitsmarktpolitik in der sozialen Marktwirtschaft. Vom Arbeitsfördergesetz zum Sozialgesetzbuch II und III, 2. Aufl., Wiesbaden, S. 126-142

Burns, T. (1961): Micropolitics: Mechanism of Institutional Change, in: Administrative Science Quarterly, 6 (3), S. 257-281

Hackenbroch, R. (1998): Verbände und Massenmedien. Öffentlichkeitsarbeit und ihre Resonanz in den Massenmedien, Wiesbaden

Höfer, M. A. (2010): Externalisierung von Reformkommunikation, in: Hoffjann, O./Stahl, R. (Hrsg.): Handbuch Verbandskommunikation, Wiesbaden S. 399-406

Hoffjann, O./Stahl, R. (Hrsg.) (2010): Handbuch Verbandskommunikation, Wiesbaden

Höpner, M. (2007): Ist Politik gegen die Verbände möglich? Mancur Olsons "the Rise and the Decline of Nations", in: Leviathan 35 (3), S. 310-347 Hoffjann, O. (2010): Verbandskommunikation und Kommunikationsmanagement: eine systemtheoretische Perspektive, in: Hoffjahn, O./Stahl, R.,

a.a.O.,S. 59-80

Hoffmann, J./Steiner, A./Vogel, M. (2007): Moderne Public Affairs versus traditionelle Interessenvertretung? Agenturen, Unternehmen und Verbände der politischen Kommunikation, in: Österreichische Zeitschrift für Politikwissenschaft (ÖZP) 36 (4), S. 425-444

Klenk, T./Weyrauch, Ph./Haarmann, A./Nullmeier, F. (2012): Abkehr vom Korporatismus? Der Wandel der Sozialversicherungen im europäischen Vergleich, Frankfurt

Koch-Baumgarten, S. (2010): Verbände zwischen Öffentlichkeit, Medien und Politik, in: Hoffjann, O./Stahl, R., a.a.O., S. 239-258

Krickhan, Th. (2010): Mittelstandsverbände in Deutschland, in: Schroeder, W./ Weßels, B., a.a.O., S. 85-104

Lang, A./Schneider, V. (2007): Wirtschaftsverbände. Verbandspolitik im Spannungsfeld von divergierenden Interessen und hierarchischer Integration, in:
Winter, Th. von/Willens, U. (Hrsg.): Interessenverbände in Deutschland, Wiesbaden, S. 221-243

Leckebusch, R. (1966): Entstehung und Wandlung der Zielsetzungen, der Struktur und der Wirkungen von Arbeitgeberverbänden, Berlin

Münkler, H./Straßenberger, G./Bohlender, M. (Hrsg.) (2006): Deutschlands Eliten im Wandel, Frankfurt

Paster, Th. (2010): Die Rolle der Arbeitgeber in der Sozialpolitik, in: Schroeder, W./Weßels, B., a.a.O., S. 342-362

Neuberger, O. (1995): Mikropolitik. Der alltägliche Aufbau und Einsatz von Macht in Organisationen, Stuttgart

Preusse, J./Zielmann, S. (2010): Verbands-PR und Lobbying im Vergleich, in: Hoffjann, O./Stahl, R., a.a.O., S. 333-352

Priddat, B./Speth R. (2007): Das neue Lobbying der Unternehmen: Public Affairs: Hans-Böckler-Stiftung, Arbeitspapier 145, Düsseldorf

Schroeder, W./Weßels, B. (Hrsg.) (2010): Handbuch Arbeitgeber- und Wirtschaftsverbände in Deutschland, Wiesbaden

Siedentopp, J. (2010): Public Affairs-Management von Großunternehmen, Münster

Speth, R. (2004): Die politischen Strategien der Initiative Neue Soziale Marktwirtschaft, Düsseldorf: Hans Böckler Stiftung, Arbeitspapier 96

Speth, R. (2006a): Lobbyismus als Elitenintegration? Von Interessenvertretung zu Public Affairs-Strategien, in: Münkler, H./Straßenberger, G./Bohlender, M., a.a.O., S. $221-236$

Speth, R. (2006b): Die zweite Welle der Wirtschaftskampagnen. Von „Du bist Deutschland bis zur Stiftung Marktwirtschaft": Arbeitspapier der Hans-BöcklerStiftung 127, Düsseldorf

Speth, R. (2010a): Arbeitgeber- und Wirtschaftsverbände in Politik und Gesellschaft, in: Schroeder, W./Weßels, B., a.a.O., S. 260-279

Speth, R. (2010b): Das Bezugssystem Politik - Lobby - Öffentlichkeit, in: Aus Politik und Zeitgeschichte 19/2010, S. 9-15

Speth, R. (2010c): Grenzen der politischen Kommunikation von Unternehmensverbänden, in: Schroeder, W./Weßels, B., a.a.O., S. 220-233

Steiner, A./Jarren, O. (2009): Intermediäre Organisationen unter Medieneinfluss? Zum Wandel der politischen Kommunikation von Parteien, Verbänden und Bewegungen, in: Marcinkowski, F./Pfetsch, B. (Hrsg.): Politik in der Mediengesellschaft (PVS-Sonderheft 42), S. 251-269

Streeck, W. (2006): Nach dem Korporatismus: Neue Eliten, neue Konflikte, in: Münkler et al., a.a.O., S. 149-175

Trampusch, Ch. (2005): Sozialpolitik in Post-Hartz-Germany, in: Welt-Trends 47, S. $77-90$

Trampusch, Ch. (2009): Der erschöpfte Sozialstaat. Transformation eines Politikfeldes, Frankfurt

Töller, A.E. (2008): Mythen und Methoden. Zur Messung der Europäisierung der Gesetzgebung des Deutschen Bundestages jenseits des 80 \%-Mythos, in Zeitschrift für Parlamentsfragen 39 (1), S. 3-17

Urban, H.J.(2010): Wohlfahrtsstaat und Gewerkschaftsmacht im FinanzmarktKapitalismus. Der Fall Deutschland, in: WSI-Mitteilungen 63 (9), S. 443-450, http://www.boeckler.de/wsimit_2010_09_urban.pdf

Vitols, S. (2006): Das "deutsche Modell“ in der politischen Ökonomie, in: Berg hahn, V. R./,Vitols, S. (Hrsg.): Gibt es einen deutschen Kapitalismus? Traditionen und globale Perspektiven der sozialen Marktwirtschaft, Frankfurt, S. 44-59 Weitbrecht, H. (2010): Arbeitgeberverbände in der Tarifpolitik und im tarifpolitischen System der Bundesrepublik, in: Schroeder, W./Weßels, B., a.a.O., S. $316-341$

Winter, Th. von (2003): Vom Korporatismus zum Lobbyismus. Forschungsstand und politische Realität, in: Forschungsjournal Neue Soziale Bewegungen 1 (3), S. $37-44$

\section{AUTOR}

RUDOLF SPETH, Dr., ist vertretender Fachbereichsleiter für den Bereich Politisches System/Staatlichkeit im Wandel an der Universität Kassel. Arbeitsschwerpunkte: Interessenvertretung, Lobbying, politische Kommunikation, Zivilgesellschaft.

rudolf.speth@uni-kassel.de 